\title{
Disaster Prevention Integrated into Commonly Used Web Rendered Systems with GIS Capabilities
}

\author{
M. Cioca, S.C. Buraga, C. Cioranu
}

\begin{abstract}
Marius Cioca
Lucian Blaga University of Sibiu

E-mail: marius.cioca@ulbsibiu.ro
\end{abstract}

\section{Sabin-Corneliu Buraga}

A. I Cuza University of Iasi

E-mail: busaco@infoiasi.ro

\section{Cosmin Cioranu}

UEFISCDI

E-mail: cosmin.cioranu@gmail.com

\begin{abstract}
The end of the 20th century brought a remarkable increase in the field of positioning techniques and communications, making them visible and available to the public, which led to an unprecedented interconnectivity. At the same time, disasters are part of our life. Regardless of their nature, measures can be taken, in order to prevent and mitigate their effects, by anticipative preparation or by avoiding the calamity area (if possible). To this end, this paper presents an integrated system, composed of a software component, a hardware component, and a decision-making human element, all having the declared role of diminishing or eliminating human and material losses.
\end{abstract}

Keywords: : DSS, Disaster, Open Layers, Apache.

\section{Introduction}

The end of the 20th century brought a remarkable increase in the field of positioning techniques and communications, making them visible and available to the public. Among them, one of the most spectacular - which revolutionized the way in which we relate to our planet was in fact the introduction of GIS (Geographical Information System) positioning technologies on the consumer level, which were actively supported by communities of developers and which transposed this knowledge into a format that the ordinary user can access, starting with the mobile ones and ending with the old and well-known PC. (e.g. Google Earth) [16]. Fire, floods, earthquakes, storms, disasters caused by human negligence can occur and can give rise to irremediable damages, but in some cases, material and human losses can be avoided or, in the worst case, diminished through a set of measures that can be assisted or implemented by means of an information system with spatial capabilities, that collects data from different sources in order to develop a prevention system for such events, that finally has an dissemination role, that can act through deployment of resources, so that the event or the events concerned affect the normal course of life as little as possible [18].

\section{Decision Support Technologies and Tools}

The complexity of sustainable development requires rational decision-making, and decisionmaking becomes increasingly difficult especially regarding environmental issues. The progress in decision-making theory and decision support systems have determined the emergence of methods 
and tools that can assist the decision-maker in making the most adequate decisions. However, in helping decision-making on complex issues, these tools are not easily developed and built [1], [7], [8], [10], [11] and [12] . The use of general accepted techniques and methods in DSSs may improve the identification and prevention of complex risk situations. These imply making strategic decisions supported by expert boards and groups. Their activity is often burdened with physical, time and cognitive barriers. Moreover, these methods and techniques used by DSSs represent only general approaching instructions on specific decision-making situations. In practice, in order to be used efficiently, they need reinterpretations, refinements, adjustments and completions.

\section{Technical Approach}

To this end, this paper presents an integrated system (fig.1), informationally and spatially scalable from a community to a certain area, that stratifies both decisional and informational passive and active elements, in order to prevent human losses and/or danger situations, generated by natural or human calamities (floods, radiations, earthquakes etc.). For harmonization, these elements have been grouped into passive elements, meaning those elements that provide information related to environment, active elements, which in this case, are human decisionmakers, that have as primary attributes the position in latitude and longitude system, as well as their type. Among the active elements of the system, we mention a list of spatially-localized decisional personnel, attached to each regional entity (village, commune, county), that can be alerted when the system detects a case of disaster, through specific algorithms. One of the main elements, of a great importance, are the specific approved sensors, both fixed and mobile, placed on the entire area of focus, that measure events like humidity level, landslides, earthquakes, radiations etc. These are equipped with point-to-point communication equipment like GSM, radio or other methods that ensure information transmission to the decisional cluster. Another passive element that brings a better accuracy to the system are the meteorologic maps, but which imply a high data volume, meaning that these should be served to the system in a semi-processed manner. The major problem of this system was the link between these elements, their harmonization being accomplished by integrating these data into a decision-making neural network, which concentrates the spatial information by a number of minimization operations, so that the result can be transposed into a GIS capable system and that generates a well-determined spatial answer. In such an approach, methodologies for this kind of wireless sensor-systems have to be used [4], security protocols for wireless sensor networks [2] have to be considered, as well as efficient algorithms, specific to this kind of mobile sensor networks [3].

\section{Computer System Implementation}

From the informational standpoint, the system is based on a web-type, client-server architecture, one of the most frequently employed in application development on one hand and sensor and meta-data set on the other hand. This approach ensures the separation of the function logic model of the application into smaller functional units, the usage being split into two major components: client and server. On the client-side, the users, both on administration level and also as common users, integrate browsing technologies, being compatible with 99,3\% [17] of all users, using technologies such as Web2.0, HTML, CSS, JavaScript, OpenLayers. At server level, Apache, PHP, Python, Inkscape, ImageMagick, GDAL, MySQL are among the technologies employed [14]. Additionally, besides these technologies, the data server receives inputs on other communication channels such as GSM or radio. The architecture used and shown in figure 2 is 


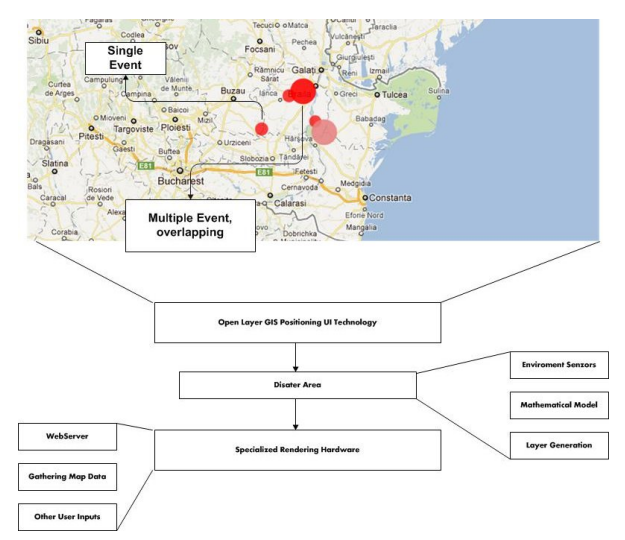

Figure 1: Integrated and scalable spatial information system

easy to approach. Likewise, the data central system can send alerts to some decision-making entities, firefighters, the police, responsible with disaster prevention and management etc. This architecture is one of the most versatile and can be accessed from: smartphones, desktop PC, laptops, tablets etc.

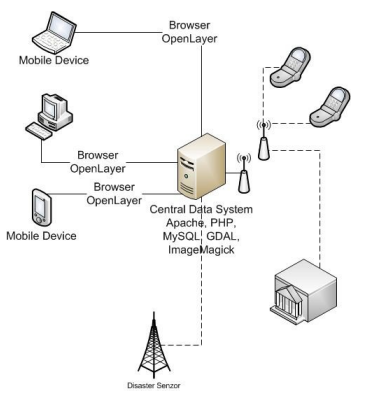

Figure 2: General system architecture

The presented system has a temporal role, giving the possibility to be loaded with statistical data. Once mature, it can play a crucial role in further cases.

From a functional standpoint, the system is divided into several distinct components:

a. the transfer component, communication; b. the alert component; c. collection hardware components passive elements, i.e. sensors, that collect states of the environment, processing systems etc.; d. Software components, i.e. the implementation of a mathematical model and a logic for the analysis of the risk of the various inputs from the external environment, all marked and positioned, using a GIS system; e. Finally, human components active, decision-making elements, which will be covered in this study only as constitutive element.

From the communication standpoint, two ways are used, namely:

a. GSM serial communication, usually for timely communications, status data or alert information, characterized by small data volume; b. or other TCP/IP wireless channels, for viewing and creating a graphical information map.

The advantage of GSM communications consists in a broader coverage area, but with a lower bandwidth, usually serial communications. They usually meet the need of speed and coverage for stationary sensors, that send the useful data in burst mode. The second is the wireless channel, which provides a wider bandwidth, but which is limited to a shorter distance. In terms of alert, 
this system integrates information capabilities of the decision-making elements, by using the following channels:

a. GSM channels, by SMS [5]; b. Ordinary channels, mail, newsletters, form-boards etc.

Industrial GSM modems ensure communication with the GSM network, but for a higher number of SMS messages, one can choose the SMS Bulk service of a local mobile network provider, which is a service provided by local mobile network operators, by which they ensure a way of interconnection of the local system with the mobile network operator. The disadvantage of such a system is the fact that this connection can be interrupted due to the way of implementation (cables), but can provide a greater number of alert messages even greater than a GSM Modem (which is, a mobile phone with advanced features of interconnectivity and interoperability with a computer) [6]. In terms of hardware component, the architecture is composed of sensors and/or other data sources, either automatic or human, which are afterwards integrated into the software architectural level. The internal structure of the software component is divided into the following (figure 3), in a top-dow approach:

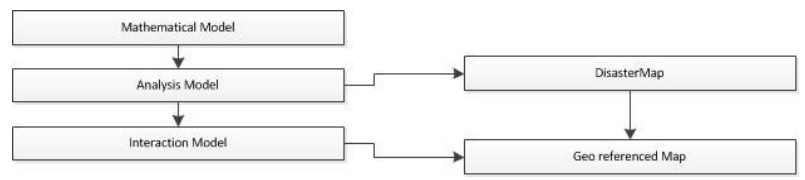

Figure 3: The internal structure of the software component

a. the mathematical model; b. the logic analysis model; c. the interaction model.

From the functional standpoint, this component uses accumulated data and by specific algorithms, it generates visually usable data, which can also be used in the analysis process.

The mathematical model has the following formal structure:

$$
S=R x R
$$

where S - geographical space, georeferenced WGS84 (as a rule) [16], but it can be transformed by using other utilities (e.g. GDAL) in any format compatible with OpenLayers [14].

$$
P_{i}(\alpha, \beta) \in \mathrm{S}
$$

The sensor coordinates, where $\alpha, \beta$, geographic coordinates

$$
V_{P_{i}(\alpha, \beta)} \in\left[\mathrm{V}_{\mathrm{pmin}}, \mathrm{V}_{\mathrm{pmax}}\right]
$$

where $V_{p \min }, V_{\text {pmax }}$ are the normal values. Above these values, maximum or minimum, a danger or disaster impact is to be generated.

Therefore, attached to each point (sensor) defined as such, the notion of impact is a specific function, defined as follows:

$$
I_{P_{i}}=0
$$

if $V_{P_{i}(\alpha, \beta)} \in\left[\mathrm{V}_{\mathrm{pmin}}, \mathrm{V}_{\mathrm{pmax}}\right]$.

$$
I_{P_{i}}=\Psi\left(V_{P_{i}(\alpha, \beta)}\right)
$$

if $V_{P_{i}(\alpha, \beta)} \notin\left[\mathrm{V}_{\text {pmin }}, \mathrm{V}_{\text {pmax }}\right]$.

and 
$\Psi \in R x R$

In order to establish the implementation of thei $\Psi$, function, the following criteria will be taken into account:

- Risk level: Disaster, High risk, Medium risk, Low risk and Minor risk.

- Event type with human implications: Earthquake, Floods and Fire.

Taking into account the above-mentioned relations, we can define the disaster area, passing to an iteration through all the points where information can be collected, generating a layer shown in Figure 4, which overlapping with the already existing layers, generates figure 1.

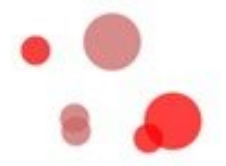

Figure 4: The layer generated on the basis of the information received from the collection points

In its turn, the logic model integrates analysis and decision-making components and prepares the needed elements for decision. At this level, one must specify the mathematical model that underlies the entire software component and has as a result a georeferenced layer, which includes the information of each important element that provides information about the environment.

The human element plays two roles, one is to be informed, namely the decision-making element, and the second is to inform oneself, in order to avoid a calamity area.

\section{Technologies}

Client [19], [14] - client requires a minimum number of software applications, which as we can see, are relatively inexpensive: a. Web Browser (Firefox, Internet Explorer >5.5, Nescape) to access the system residing on the server; b. Access to the server that hosts the system (Intranet, Internet) to ensure the interconnectivity for this station. Any communication media can usually be employed, from wire to wireless technologies; c. The required hardware is restricted to the above-mentioned software requirements; d. For interface software, Open Layer is used (in order to render the data).

Server [14] - the server software requirements are the following: a. Operation system: Linux Based -provides very good stability in terms of security and performance; b. Programming language: PHP 5.x; c. SGBD: MySQL 5.x; d. Web Server WEB : Apache 2.x; e. GDAL and ImageMagick for data display.

\section{The Experimental Section. Results}

The methodology for impact assessment is:

a. Establishing the area center or sensor coordinates; b. Establishing the limits affected on the 4 major directions $\mathrm{N}, \mathrm{S}, \mathrm{E}, \mathrm{W}$; c. Establishing the negative impact mitigation rates $T_{N}, T_{S}$, $T_{E}, T_{V}$

In order to calculate distance D between two coordinate points P1(lat 1, long $\left._{1}\right)$ and P2(lat 2 , long $\left._{2}\right)$ ) the haversine formula was used: [15], [20] 


$$
D=R * 2 * \arcsin \left(\sqrt{\sin ^{2}\left(\frac{\Delta l a t}{2}\right)+\cos \left(l_{a t}\right) * \cos \left(\operatorname{lat}_{2}\right) * \sin ^{2}\left(\frac{\Delta l o n g}{2}\right)}\right)
$$

where $\mathrm{R}$ is the Earth radius, which varies from the Equator to the Poles between 6378.14 and 6356.78 , where the accuracy of the above-mentioned formula derives from, an error of about $0.5 \%$.

Stage 1

Collecting the field data following the format below (in order to calculate distance D1).

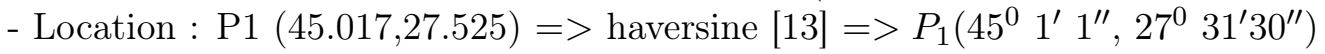

- Affected area

o Maximum coordinates: (N (45.097, 27.557), E (45.056, 27.613), S(45.012, 27.552), $\mathrm{V}(45.053,27.482)$;

o Meters, from the point of origin [16]: N 5088m, with an impact lasting for up to 8 days, $\mathrm{E} 4661 \mathrm{~m}, 6$ days, $\mathrm{S} 4449 \mathrm{~m}, 5$ days, $\mathrm{V}$ 5792m, 8 days

- Impact parameters are indicated at each point.

Collecting the field data following the format below (in order to calculate the distance D2).

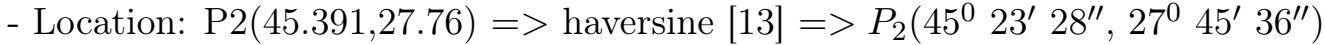

- Affected area

o Coordinates: N(45.415, 27.757), E(45.395,27.783), S(45.375,27.757), V(45.395,27.783)

o Meters, from the point of origin: N 2178m, with an impact up to 6 days, E1897m, 6 days, $\mathrm{S} 1992 \mathrm{~m}, 8$ days, $\mathrm{V} 2136 \mathrm{~m}, 9$ days

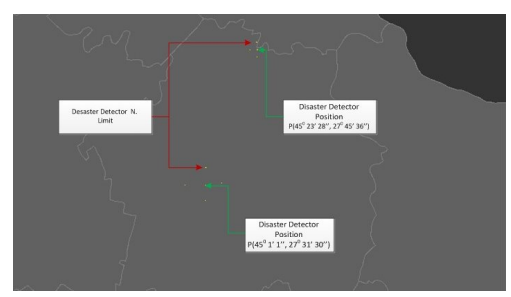

Figure 5: Disaster positioning on the map

\section{Stage 2}

Stage 2 involves the development of the graphic management functions.

The progression calculation function for the affected area

$$
D(t)=\frac{t_{\max }-t}{t_{\max }} d_{\max }
$$

where $t \in\left[0, t_{\max }\right], d \in\left[0, d_{\max }\right]$

$$
D(t) \in\left[0, d_{\max }\right]
$$

The impact function, calculates the impact level using the maximum distance, being applied on the 4 directions $\mathrm{N}, \mathrm{E}, \mathrm{S}, \mathrm{W}$

$$
l(t, d)=\frac{t_{\max }-t}{t_{\max }} \frac{d}{d_{\max }}
$$

where $t \in\left[0, t_{\max }\right], d \in\left[0, d_{\max }\right]$

$$
l(t, d) \in[0,1]
$$


where 0 means there is no impact and 1 , maximum impact.

Figure 6 results using the relations [2], [4]

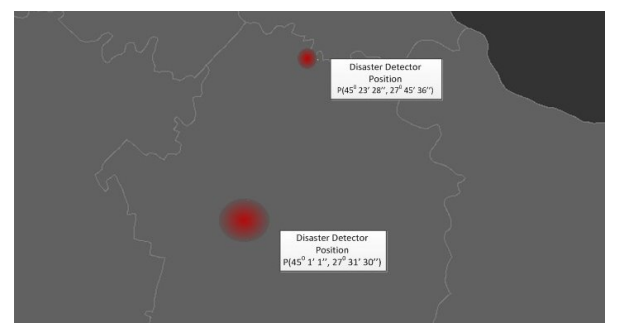

Figure 6: Disaster size at moment $\mathrm{T}_{0}=0$

The following figure represented in a logarithmic scale disaster cases, using the $\mathrm{N}$ direction.

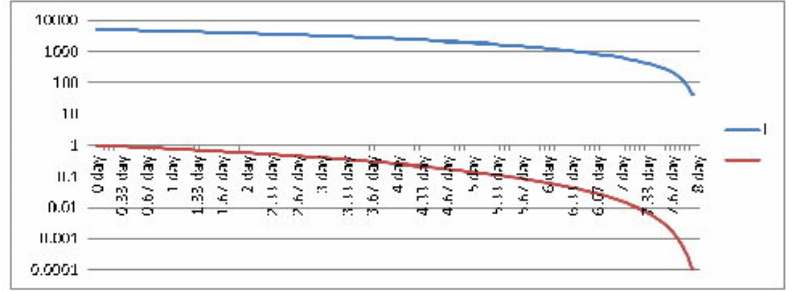

Figure 7: Represented in a logarithmic scale where: blue is space variation/day affected on direction $\mathrm{N}$; red is intensity variation of the space affected / day on direction $\mathrm{N}$

On zero-day of disaster the area was about $5000 \mathrm{~m}$ and the eighth day this impact falls to 0 .

On zero-day of the impact the disaster was labeled 1 then as time passed by, on the eighth day, it fell to 0 .

The same calculation is used for the impact area $\mathrm{P} 1$ for the eastern element.

\section{Conclusions and Future Research}

In conclusion, we can say that, in order to develop high-quality and complex DSSs, able to cover as much as possible in case of disasters, on the one hand, a multidisciplinary approach is required, an approach which should bring together specialists in various fields, such as: environment, GIS, geographers, mathematicians, computer scientists, organizations in charge with such situations etc.; and on the other hand, a global approach which should reunite institutions and people from different countries, as such phenomena are not restricted to the borders of one specific country and they may affect large areas, encompassing people and goods from different states and at the same time, the transfer of know-how between partners/researchers from different countries brings benefits to such catastrophic situations, that might end up with material damages or worse, with the loss of human lives.

\section{Bibliography}

[1] Anica-Popa I., Cucui G., A Framework for Enhancing Competitive Intelligence Capabilities using Decision Support System based on Web Mining Techniques, INT J COMPUT COMMUN, ISSN 1841-9836, 4(4):326-334, 2009. 
[2] Aseri T.C., Singla N., Enhanced Security Protocol in Wireless Sensor Networks, INT J COMPUT COMMUN, ISSN 1841-9836, 6(2):214-221, 2011.

[3] Ban D., Yang W., Jiang J., Wen J., Dou W., Energy-Efficient Algorithms for k-Barrier Coverage In Mobile Sensor Networks, INT J COMPUT COMMUN, ISSN 1841-9836, 5(5):616$624,2010$.

[4] Chen J.I.-Z. , Chung Y.-N., A Data Fusion Methodology for Wireless Sensor Systems, INT J COMPUT COMMUN, ISSN 1841-9836, 7(1):39-52, 2012.

[5] Cioca, M., Cioca, L.I. Decision Support Systems used in Disaster Management, in Decision Support Systems, Chiang S. Jao (Ed.), ISBN: 978-953-7619-64-0, 2010.

[6] Cioca, M., Cioca, L.I., Buraga, S.C., Spatial (Elements) Decision Support System Used in Disaster Management, Digital EcoSystems and Technologies Conference, IEEE-DEST, ISBN: 1-4244-0467-3, IEEE Catalog Number: 07EX1418C, pp. 607-612, 2007.

[7] Filip, F.G. Decision Support and Control for Large-Scale Complex Systems, Annual Reviews in Control (Elsevier),ISSN: 1367-5788, 32(1): 61-70, 2008.

[8] Filip, F.G. Sisteme suport pentru decizii, ISBN 978-973-31-2308-8, Editura Tehnica, Bucuresti, 2007.

[9] Peng, Y., Gang Kou, Shi, Y., and Chen, Z., A Descriptive Framework for the Field of Data Mining and Knowledge Discover, Int. J. of Information Technology 85 Decision Making, 7(4):639-682, 2008.

[10] Peng, Y., Gang Kou, Wang, G., Wu, W., and Shi, Y., Ensemble of software defect predictors: an AHP-based evaluation method, DOI: 10.1142/S0219622011004282, Int. J. of Information Technology \& Decision Making, 10(1):187-206, 2011.

[11] Power, D. J. Decision support systems: Concepts and Resources for Managers, Quorum Books, Westport, Connecticut, 2002.

[12] Suduc-Ana Maria, Bizoi, M., Filip, F.G., User Awareness about Information Systems Usability, STUDIES IN INFORMATICS AND CONTROL, 19(2):145-152, 2010.

[13] http://andrew.hedges.name/experiments/convert_lat_long/

[14] http://asrc.ro/imeteosat_beta (accessed on 07.04.2012)

[15] http://en.wikipedia.org/wiki/Haversine_formula

[16] http://support.google.com/earth/bin/answer.py?hl=ro\&answer=148110 (accesed on 2012)

[17] http://www.articlesnatch.com/Article/Certification-Authorities-With-Browser-UbiquityOf-99-3-Are-Best-In-Industry-/253347

[18] http://www.cimec.ro/Resurse/Patrimoniu/Dezastre.htm (accessed on 5.04.2012)

[19] http://www.developer.com/java/web/print.php/10935_3528381_2 (accessed on 3.04.2012)

[20] http://www.movable-type.co.uk/scripts/gis-faq-5.1.html 